\title{
Attitude and Its' associated factors towards Mental Illness Among Residents of Mertule Mariam Town, Northern Ethiopia: Mixed Method
}

Berhanu Yeshanew ( $\square$ berhanuyeshanew@gmail.com )

Dire Dawa Univresity https://orcid.org/0000-0003-4680-3792

Zegeye Yohannis

Addis Ababa University School of Medicine

Shegaye Shumet

University of Gondar College of Medicine and Health Sciences

Dessie Abebaw

University of Gondar College of Medicine and Health Sciences

Asmare Belete

Wollo University college of Medicine and Health science

Research

Keywords: Attitude, mental illness, Mertule Mariam, Ethiopia

Posted Date: April 23rd, 2020

DOI: https://doi.org/10.21203/rs.3.rs-23093/v1

License: (c) (1) This work is licensed under a Creative Commons Attribution 4.0 International License. Read Full License 


\section{Abstract}

Background: Mental illness affects cognition, emotion, and behavior of an individual. It accounts for $13 \%$ of the global burden of diseases. About $76 \%$ and $85 \%$ of people in low and middle-income countries with severe mental illness did not receive treatment due to fear of expected discrimination and stigma to diagnosed people with mental illness. There are no published works on the attitude of the community in this study area. This study aimed to assess the attitude and its' predictors towards mental illness among residents of Mertule Mariam town, Ethiopia 2017.

Methods: Community-based cross-sectional study was conducted from May to June 2017. Data were collected from 964 participants using Community Attitude to Mental Illness Inventory (CAMI). Data were entered using epi-info version 7 and exported to SPSS version 20. Descriptive and binary logistic regression analyses were employed to identify the associated factors to attitude.

Result: The response rate was $98.2 \%$ with the sex distribution of $58.5 \%$ (554) males. The attitudes of the community were more authoritarian $(52.8 \%)$, less benevolent $(52.3 \%)$, more socially restrictive $(38.8 \%)$ and positive to mental health service (59.2\%). Age [AOR=2.50, $\mathrm{Cl} 1.56-4.23$, P-value= 0.001], educational level, occupational status, getting information about mental illness, and knowing someone who has mental illness have been significantly associated to authoritarianism. Age (18-24) [AOR=3.08(1.5-6.3) pvalue $=0.002]$, educational status, occupational status and getting information about mental illness were the significant variables for benevolence subscale. Age, marital status, getting information about mental illness [AOR=0.02, $\mathrm{Cl}$ 0.05-0.75, P-value $<0.05]$, sources of information were significant variables for social restrictiveness subscale. Marital status, occupational status, history of mental illness, relationship with mentally ill, and involved in caring for mentally ill [1.85(1.28-2.65) $\left.{ }^{\star \star}\right]$ were significantly associated factors for community mental health ideology.

Conclusion and recommendation: The findings of this study showed that the attitude of residents of Mertule Mariam town was more authoritarian (undermining), less benevolent, less socially restrictive (less socially avoidant) and more positive for mental health service. The positive attitude of the participant was associated with getting information about mental illness. Using mass media and health institutions to disseminate information about mental health is essential to improve the attitude of the community.

\section{Background}

Mental illness is a condition that affects the cognition, emotion, and behavior of an individual. It also affects the individual's ability to realize his potential, cope with normal life stressors, act productively and contribute to his community $(1,2)$. It is one of the major contributors of disability-adjusted life of years(DALYs) and the global burden of disease $(\mathrm{GBD})(3,4)$.

In Ethiopia, mental illness is the leading contributor to disease burden out of non-communicable diseases (NCD) in which it comprised $11 \%$ exceeding HIV/AIDS (5). Even though mental illnesses are highly prevalent and incapacitating to the community of this world (6), no more help is requested from the 
modern health facility. The community's attitude hinders the help-seeking intention of the community to mental illness (7).

Larger proportions of people with mental illness never receive treatment from mental health care staff globally. Similarly, more than three-fourths of people with mental illness in low and middle-income countries never receive treatment. This is because people with mental illness fear stigma and discrimination from the community $(1,8)$.

Nigerian community has widely held bad attitude believing that mentally ill patients are dangerous due to their violent behaviors including avoiding basic social contacts; the majority will be afraid to have a conversation with people with mental illness and only a few segments of the community will consider having marriage consideration with them(9).

Community-based studies illustrated that the community rejects marriage with people with mental illness, isolate and put in separate place under supervision. Most of the respondents afraid to be neighbors, feel ashamed to talk about someone who has a mental illness that lives in the home together with them and refused to maintain the friendship. The community associated mental illness with difficulty at work and lack of will power and discipline (10-13).

Most of the Ethiopian community believes that mental illnesses are to be caused by "punishing hands of God" for disobeying the principles, doctrines and social taboos (14). For instance, in the Borana seminomadic population, the majority of the community believes supernatural influences cause mental illness. Bewitchment, witchcraft, possession by evil spirits were the central causes not only for " madness" but also for property loss. People left someone who has mental illness alone if he/she did not respond with treatment, and based on patient condition, he/she may choose to live with the family getting his/her basic needs or wander around the street naked (15).

A study done in Nigeria states that being male and literate are the factors associated with a positive attitude to mental illness. Literates have 7 times more positive attitudes to mental illness than their counters (9). Based on a study done in Ghana, females are more authoritarian than men are and social restrictiveness decreases with age. More educated people are less authoritarian and less socially restrictive than persons with only basic education are. They also expressed more benevolent than less educated (16). Females have a bad attitude on marital prospects with mentally ill and illiterates have a more negative attitude to live with people who have a mental illness than literates (17).

In southwest Ethiopia, a study showed higher educational status and exposure to mental illness information decreases stigma (14). Community-based cross-sectional study done in Worabe town showed that illiterates have a more socially restrictive and less humanistic attitude. Having mental health information is highly associated with less socially restrictive and a less authoritarian attitude towards people with mental illness (18). 
Even though there are few studies in Ethiopia $(12,14,15,17-21)$ assessing the attitude of the community to mental illness but they are limited to the southern and western regions. As much as we know, there are no published data regarding the attitude of the community to mental illness in the northern part of Ethiopia. Therefore, this study has the importance of determining the attitude of the community to mental illness among residents of Mertule Mariam town, Northern Ethiopia, Ethiopia.

\section{Methods}

Study design and setting: Community-based cross-sectional study design using mixed (both qualitative and quantitative) methods were conducted in Mertule Mariam town, East Gojam Zone, Ethiopia from May to June 2017.

\section{Study population}

All residents of the town aged 18 years or more and those who reside 06 months and more were included. Those who were severely ill and unable to communicate verbally were excluded from the study.

\section{Sampling Procedure and Technique}

Multistage sampling technique was applied. The sampled households were distributed to the two kebelles proportionally. The households were selected with a systematic random sampling method considering that they are homogeneous. The first household, and when there was more than one adult in each selected household, was selected using a lottery method. Using double population proportion formula, we estimate sample size for associated factors and we got 964 with a $10 \%$ non-response rate.

Data collection tools: Data were collected using interviewer-administered semi-structured questionnaire by 6 clinical nurses. The tool consists of socio-demographic, illness perception, social support and Community Attitude to Mental IIIness Inventory (CAMI) including information and exposure-related variables. CAMI has a total of 40 items with four sub-scales which are authoritarianism, benevolence, social restrictiveness, and community mental health ideology. It is five scales Likert ( $1=$ strongly disagree, 2 = disagree, 3 = neutral, 4 = agree, 5 = strongly agree). It has been used in different countries of Africa including Ethiopia $(16,22)$. It has been checked and validated in Ethiopia. The reliability Index using

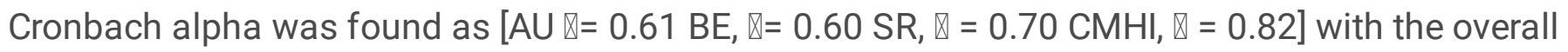
reliability of CAMI scale $a=0.84$ (18). Four focus group discussions were held that include religious leaders, health workers and community participants who were selected purposely.

Data Processing and Analysis: The collected data were entered using Epi info 7th version and exported to SPSS version 20 for analysis. Descriptive statistics and binary logistic regression were used. A P-value of less than 0.2 and a chi-square test was considered to identify factors associated with outcome variable on bivariate logistic regression. These variables were taken to multivariate logistic regression and a $\mathrm{p}$ value less than 0.05 was taken as statistically significant presented by OR with $95 \%$ confidence interval. Qualitative data were collected by the principal investigator with Amharic language and later transcribed 
to the English language. The transcribed data were organized into a theme and were presented being triangulated with quantitative data.

Ethical Consideration: Ethical clearance was obtained from the institutional review committee of the University of Gondar and Amanuel mental specialized hospital. Permission paper from the town administration was requested and distributed to the two kebelle administrations. Data collectors were explaining about the aim of the study and were asking oral consent before they started data collection. The right to ask questions about the study and to terminate the interview whenever they want to stop was offered for the study participants. The information gathered from the participants were kept confidential.

\section{Result}

\subsection{Socio-demographic characteristics}

Among the 964 study participants, 947 responded to the interview completely which make $98.23 \%$ response rate. out of the respondents, 58.5\% (554) were males while 393 (41.5\%) were females (Table1). Amhara ethnicity (99.7\%) and orthodox religion (98.7\%) took a greater proportion of the respondents. Only $18.2 \%$ (178) of respondents have a degree and above educational level, while $12.2 \%$ of respondents cannot read and write (Table 1). 
Table 1

socio-demographic distributions of participants among residents of Mertule Mariam town, Northern Ethiopia, Ethiopia, 2017.

\begin{tabular}{|c|c|c|c|}
\hline Variables & Category & Frequency & percent \\
\hline \multirow[t]{4}{*}{ Age } & $18-24$ & 241 & 25.4 \\
\hline & $25-34$ & 463 & 48.9 \\
\hline & $35-44$ & 193 & 20.4 \\
\hline & $>44$ & 50 & 5.3 \\
\hline \multirow[t]{2}{*}{ Sex } & male & 554 & 58.5 \\
\hline & female & 393 & 41.5 \\
\hline \multirow[t]{4}{*}{ marital status } & single & 426 & 45.0 \\
\hline & married & 417 & 44.0 \\
\hline & divorced & 85 & 9.0 \\
\hline & widowed & 19 & 2.0 \\
\hline \multirow[t]{2}{*}{ Ethnicity } & Amhara & 944 & 99.7 \\
\hline & other & 3 & .3 \\
\hline \multirow[t]{2}{*}{ Religion } & orthodox & 935 & 98.7 \\
\hline & other(Muslim and protestant) & 12 & 1.3 \\
\hline \multirow[t]{5}{*}{ Educational Status } & unable to read and write & 116 & 12.2 \\
\hline & elementary school & 126 & 13.3 \\
\hline & secondary school & 260 & 27.5 \\
\hline & college diploma & 267 & 28.2 \\
\hline & degree and above & 178 & 18.8 \\
\hline \multirow{7}{*}{$\begin{array}{l}\text { Occupational } \\
\text { Status }\end{array}$} & government employee & 387 & 40.9 \\
\hline & housewife & 70 & 7.4 \\
\hline & farmer & 59 & 6.2 \\
\hline & NGO employee & 45 & 4.8 \\
\hline & merchant & 168 & 17.7 \\
\hline & student & 152 & 16.1 \\
\hline & other & 66 & 7.0 \\
\hline
\end{tabular}




\begin{tabular}{|llll|}
\hline Variables & Category & Frequency & percent \\
\hline Estimated monthly family Income & under extreme poverty $(<750)$ & 290 & 30.6 \\
\cline { 2 - 4 } & Under poverty $(751-1200)$ & 113 & 11.9 \\
\cline { 2 - 4 } & Above poverty level $(>1201)$ & 544 & 57.4 \\
\hline
\end{tabular}

\subsection{Respondent social support}

Among participants, only $17.2 \%$ have strong social support while $39.6 \%$ (375) have poor social support and the rest covers a moderate level of social support.

\subsection{Mental health information}

About $575(60.72 \%)$ of 947 participants have ever got information about mental illness during the last one year and $39.7 \%$ (376) of participants got the information from people other than media or health institutions while $11.6 \%$ got the mental health information from health institutions. Mass media gives information to $6.5 \%$ of participants while $4.4 \%$ of participants got the information from magazines. About $37.8 \%$ of participants got information about mental illness from school and religious institutions.

\subsection{Contacts with someone who has a mental illness}

About $69.4 \%$ of participants know someone who has a mental illness and only $6.9 \%$ of them have relatives who have a mental illness. Around $70 \%$ of respondents had never involved in caring for someone who has a mental illness. Thirty-three percent o participants have been hurt and $72.5 \%$ of participants have witnessed hurt by people who have a mental illness.

\subsection{Illness perception of mental illness}

Around $58.1 \%$ of participants graded mental illness as very sever and $81.6 \%$ of participants believe that mental illness requires treatment. Most (71.5\%) of believing different psychosocial factors as major causes for mental illness while $6.5 \%$ and $7.5 \%$ of participants believe evil spirit possession and God's punishment as a cause of mental illness respectively. 
Table 2

Perception of mental illness among residents of Mertule Mariam town, Northern Ethiopia, Ethiopia, 2017.

\begin{tabular}{|llll|}
\hline Variables & Category & frequency & percent \\
\hline Perceived severity of mental illness & mild & 24 & 2.5 \\
& moderate & 66 & 7.0 \\
\hline Perceived cause of mental illness & sever & 307 & 32.4 \\
& very sever & 550 & 58.1 \\
& psychosocial factor & 677 & 71.5 \\
\hline & nerve damage & 13 & 1.4 \\
\hline & poverty & 22 & 2.3 \\
\hline substance use & 102 & 10.8 \\
\hline Perceived need of treatment for mental illness & yes & 59 & 6.2 \\
\hline & no & 71 & 7.5 \\
\hline Perceived good place for help & God's punishment & 773 & 81.6 \\
\hline & holywater & 174 & 18.4 \\
\hline & holywater and hospital & 101 & 10.7 \\
\hline & hospital & 614 & 64.7 \\
\hline & sorcerer & 24 & 2.5 \\
\hline
\end{tabular}

\subsection{Community attitude to mental illness}

Respondents were more authoritarian (52.8\%), less benevolent $(52.3 \%)$, more socially restrictive (38.8\%) and positive to mental health service (59.2\%). Those aged greater than 44 years old show that they are more authoritarian and more socially restrictive than the other age groups $(66 \%)$. Females are more authoritarian than males with a negative attitude to mental health service and care $(55 \%, 44 \%)$. Married people are more authoritarian than singles and less authoritarian than widowed ones (58.5\%). Peoples who had information about mental illness are less socially restrictive than their counters $(63.9 \% \mathrm{Vs}$ $51.7 \%)$.

\subsection{Factors associated with community attitude toward mental illness}

Factors that have a p-value less than 0.2 on bivariate regression were taken into multivariate logistic regression and those that have a p-value less than 0.05 were taken as statistically significant.

\section{Authoritarianism}


The odds of being more authoritarian among those aged between 35-44 years old is 2.5 times more as compared to the age group 18-24 years old [ $\mathrm{Cl}=1.56-4.23, \mathrm{p}$-value $=0.001]$. The odds of being authoritarian among those who have elementary education was decreased by $60 \%$ as compared to illiterates [0.4 (0.19-0.77) p-value $=0.007]$. The odds of being authoritarian decreased by $67 \%$ among degree holders as compared to illiterates.

\section{Benevolence}

The odds of being more benevolent among peoples aged $>=44$ years old is 3 times more as compared to the reference age $(18-24)[A O R=3.08(1.5-6.3) p$-value $=0.002]$. The probability of being benevolent decreased by $83 \%$ among Peoples who perceive mental illness mild as compared with those who perceive very sever $[\mathrm{AOR}=0.17(0.06-0.5) \mathrm{p}$-value $=0.001]$.

\section{Social restrictiveness}

People aged between 35-44 years old are 2.7 times more likely to stigmatize people who have mental illness as compared to those aged between 18-24 years old [Cl (1.7-4.33), p-value $=0.001]$. Widowed peoples are prone to stigmatize mentally ill people 4 times as compared to single ones [Cl (1.17-13.7), pvalue]. Poor Knowledge of mental illness exposes to social restrictiveness by $1.75[\mathrm{Cl}(1.31-2.34)$, $\mathrm{p}$-value $=0.001]$ times as compared to having good knowledge.

\section{Community mental health ideology}

The odds of having a favourable attitude to mental health service integration among married people is $1.65[(1.16-2.36), p$-value $=0.005]$ times more as compared to single people while widowed were [6.2(1.56-24.8), $p$-value $=0.01$ times] more favorable than singles. The odds of having favorable attitude among peoples who perceive mental illness need treatment were $2.17((1.47-3.2) p$-value $<0.001)$ times more as compared to their counters. 
Table 3

Binary logistic regression analysis of respondent' selected Socio-demographic characteristics and other factors associated with attitude towards mental illness among residents of Mertule Mariam town, Northern Ethiopia, Ethiopia, 2017.

\begin{tabular}{|c|c|c|c|c|c|}
\hline \multirow[t]{2}{*}{ Variables } & \multirow[t]{2}{*}{ Category } & \multicolumn{2}{|l|}{ Authoritarianism } & \multicolumn{2}{|l|}{ Benevolence } \\
\hline & & COR & AOR & COR & AOR \\
\hline \multirow[t]{4}{*}{ Age } & $18-24$ & ref & Ref & Ref & Ref \\
\hline & $25-34$ & $1.82(1.32-2.49)$ & $\begin{array}{l}1.70(1.2- \\
2.5)^{\star \star}\end{array}$ & $\begin{array}{l}1.08(0.79- \\
1.48)\end{array}$ & $\begin{array}{l}1.10(0.80- \\
1.52)\end{array}$ \\
\hline & $35-44$ & $3.06(2.06-4.54)$ & $\begin{array}{l}2.5(1.56- \\
4.2)^{\star \star}\end{array}$ & $\begin{array}{l}1.39(0.95- \\
2.04)\end{array}$ & $\begin{array}{l}1.38(0.93- \\
2.05)\end{array}$ \\
\hline & $>44$ & $3.10(1.63-586)$ & $\begin{array}{l}2.6(1.16- \\
5.70)^{\star}\end{array}$ & $\begin{array}{l}2.26(1.2- \\
4.25)\end{array}$ & $\begin{array}{l}2.09(1.08- \\
4.06)^{\star}\end{array}$ \\
\hline \multirow[t]{5}{*}{ Educational status } & Illiterate & Ref & Ref & Ref & Ref \\
\hline & $\begin{array}{l}\text { elementary } \\
\text { school }\end{array}$ & $1.82(1.12-2.94)$ & $\begin{array}{l}0.4(0.2- \\
0.7)^{\star \star}\end{array}$ & $\begin{array}{l}0.71(0.43- \\
1.18)\end{array}$ & $\begin{array}{l}0.39(0.20- \\
0.76)^{\star \star}\end{array}$ \\
\hline & $\begin{array}{l}\text { secondary } \\
\text { school }\end{array}$ & $0.72(0.45-1.13)$ & $\begin{array}{l}0.88(0.54- \\
1.44)\end{array}$ & $\begin{array}{l}0.75(0.48- \\
1.16)\end{array}$ & $\begin{array}{l}0.93(0.58- \\
1.47)\end{array}$ \\
\hline & Diploma & $1.09(0.75-1.60)$ & $\begin{array}{l}0.4(0.21- \\
0.86)^{*}\end{array}$ & $\begin{array}{l}0.551(0.35- \\
0.85)\end{array}$ & $\begin{array}{l}0.46(0.23- \\
0.91)^{\star}\end{array}$ \\
\hline & $\begin{array}{l}\text { degree and } \\
\text { above }\end{array}$ & $1.05(0.7-1.54)$ & $\begin{array}{l}0.3(0.15- \\
0.7)^{\star \star}\end{array}$ & $\begin{array}{l}0.95(0.59- \\
1.52)\end{array}$ & $\begin{array}{l}0.36(0.17- \\
0.75)^{\star \star}\end{array}$ \\
\hline \multirow[t]{2}{*}{ Getting information } & yes & $0.82(0.63-1.07)$ & $\begin{array}{l}4.18(1.1- \\
16.2)^{\star}\end{array}$ & $\begin{array}{l}0.83(0.63- \\
1.07)\end{array}$ & $\begin{array}{l}0.22(0.06- \\
0.81)^{\star}\end{array}$ \\
\hline & no & Ref & Ref & Ref & Ref \\
\hline \multirow[t]{2}{*}{ Knowing mentally ill } & yes & $1.37(1.04-1.81)$ & $\begin{array}{l}0.64 \\
(0.45- \\
0.9)^{\star}\end{array}$ & $\begin{array}{l}0.89(0.67- \\
1.18)\end{array}$ & $\begin{array}{l}0.95(0.71- \\
1.28)\end{array}$ \\
\hline & no & Ref & Ref & Ref & Ref \\
\hline \multirow[t]{2}{*}{ hurt by mentally ill } & yes & $\begin{array}{l}1.39(1.06- \\
1.82)\end{array}$ & $\begin{array}{l}0.67(0.48- \\
0.93)^{\star}\end{array}$ & $\begin{array}{l}1.26(0.96- \\
1.66)\end{array}$ & $\begin{array}{l}0.71(0.5- \\
0.9)^{\star}\end{array}$ \\
\hline & no & Ref & Ref & Ref & Ref \\
\hline \multirow[t]{3}{*}{$\begin{array}{l}\text { Perceived severity of } \\
\text { mental illness }\end{array}$} & Mild & $1.69(0.71-4.01)$ & $\begin{array}{l}0.2(0.06- \\
0.57)^{\star \star}\end{array}$ & $\begin{array}{l}4.46(1.64- \\
12.1)\end{array}$ & $\begin{array}{l}0.17(0.06- \\
0.50) \star \star\end{array}$ \\
\hline & Moderate & $0.42(0.24-0.72)$ & $\begin{array}{l}0.4(0.17- \\
1.15)\end{array}$ & $\begin{array}{l}0.71(0.42- \\
1.21)\end{array}$ & $\begin{array}{l}0.37(0.15- \\
1.01)\end{array}$ \\
\hline & Sever & $0.97(0.73-1.28)$ & $\begin{array}{l}0.4(0.50- \\
1.04)\end{array}$ & $\begin{array}{l}1.19(0.90- \\
1.58)\end{array}$ & $\begin{array}{l}0.41(0.16- \\
1.03)\end{array}$ \\
\hline
\end{tabular}




\begin{tabular}{lllll|} 
Very sever & Ref & Ref & Ref & Ref \\
\hline
\end{tabular}

${ }^{*} \mathrm{p}<0.05{ }^{* *} \mathrm{p}<0.01$ ref $=$ reference. ${ }^{*}$ other $=$ on street, other people's home, ${ }^{* *}$ other people $=$ informed by somewhere, ${ }^{\star \star *}$ other $=$ jobless, retired and daily laborer 
Table 4

Binary logistic regression analysis of respondent' selected Socio-demographic characteristics and other factors associated with attitude towards mental illness among residents of Mertule Mariam town, Northern Ethiopia, Ethiopia, 2017.

\begin{tabular}{|c|c|c|c|c|c|}
\hline \multirow[t]{2}{*}{ variables } & \multirow[t]{2}{*}{ Category } & \multicolumn{2}{|c|}{ social restrictiveness } & \multicolumn{2}{|c|}{$\begin{array}{l}\text { community mental health } \\
\text { ideology }\end{array}$} \\
\hline & & $\begin{array}{l}\text { Crude Odd } \\
\text { Ratio }\end{array}$ & Adjusted OR & $\begin{array}{l}\text { Crude Odd } \\
\text { Ratio }\end{array}$ & Adjusted OR \\
\hline \multirow[t]{4}{*}{ Age } & $18-24$ & Ref & Ref & Ref & Ref \\
\hline & $25-34$ & $\begin{array}{l}0.77(0.56- \\
1.07)\end{array}$ & $\begin{array}{l}1.7(1.2- \\
2.42)^{\star \star}\end{array}$ & $\begin{array}{l}0.77(0.56- \\
1.05)\end{array}$ & $\begin{array}{l}0.74(0.51- \\
1.08)\end{array}$ \\
\hline & $35-44$ & $\begin{array}{l}0.92(0.63- \\
1.36)\end{array}$ & $\begin{array}{l}2.7(1.7- \\
4.33)^{\star \star}\end{array}$ & $\begin{array}{l}0.89(0.6- \\
1.31)\end{array}$ & $\begin{array}{l}0.91(0.55- \\
1.48)\end{array}$ \\
\hline & $>44$ & $\begin{array}{l}2.83(1.49- \\
5.36)\end{array}$ & $\begin{array}{l}2.3(1.1- \\
4.76)^{\star}\end{array}$ & $\begin{array}{l}1.72(0.87- \\
3.42)\end{array}$ & $\begin{array}{l}0.95(0.42- \\
2.17)\end{array}$ \\
\hline \multirow[t]{4}{*}{ marital status } & Single & Ref & Ref & Ref & Ref \\
\hline & Married & $\begin{array}{l}1.38(1.05- \\
1.83)\end{array}$ & $\begin{array}{l}1.28(0.92- \\
1.77)\end{array}$ & $\begin{array}{l}1.53(1.15- \\
2.01)\end{array}$ & $\begin{array}{l}1.65(1.16- \\
2.36)^{\star \star}\end{array}$ \\
\hline & Divorced & $\begin{array}{l}0.87(0.53- \\
1.44)\end{array}$ & $\begin{array}{l}0.81(0.47- \\
1.41)\end{array}$ & $\begin{array}{l}0.53(0.33- \\
0.85\end{array}$ & $\begin{array}{l}0.69(0.40- \\
1.19)\end{array}$ \\
\hline & Widowed & $\begin{array}{l}7.04(2.29- \\
21.6)\end{array}$ & $\begin{array}{l}3.75(1.1- \\
12.67)^{\star}\end{array}$ & $\begin{array}{l}4.25(1.22- \\
14.8)\end{array}$ & $\begin{array}{l}6.2(1.56- \\
24.8)^{\star}\end{array}$ \\
\hline \multirow{7}{*}{$\begin{array}{l}\text { Occupational } \\
\text { status }\end{array}$} & Gov't employee & Ref & Ref & Ref & Ref \\
\hline & Housewife & $\begin{array}{l}1.45(0.86- \\
2.4)\end{array}$ & $\begin{array}{l}1.45(0.82- \\
2.56)\end{array}$ & $\begin{array}{l}0.41(0.24- \\
0.69)\end{array}$ & $\begin{array}{l}0.45(0.21- \\
0.95)^{\star}\end{array}$ \\
\hline & Farmer & $\begin{array}{l}1.5(0.88- \\
2.67)\end{array}$ & $\begin{array}{l}1.12(0.61- \\
2.07)\end{array}$ & $\begin{array}{l}0.45(0.25- \\
0.78)\end{array}$ & $\begin{array}{l}0.30(0.13- \\
0.71)^{\star \star}\end{array}$ \\
\hline & NGO employee & $\begin{array}{l}1.1(0.58- \\
2.1)\end{array}$ & $\begin{array}{l}0.92(0.46- \\
1.83)\end{array}$ & $\begin{array}{l}0.48(0.26- \\
0.90\end{array}$ & $\begin{array}{l}0.31(0.15- \\
0.67)^{\star \star}\end{array}$ \\
\hline & Merchant & $\begin{array}{l}1.37(0.94- \\
1.98)\end{array}$ & $\begin{array}{l}1.34(0.90- \\
2.01)\end{array}$ & $\begin{array}{l}0.53(0.37- \\
0.77)\end{array}$ & $\begin{array}{l}0.41(0.25- \\
0.69)^{\star \star}\end{array}$ \\
\hline & Student & $\begin{array}{l}0.94(0.64- \\
1.40)\end{array}$ & $\begin{array}{l}0.91(0.58- \\
1.43)\end{array}$ & $\begin{array}{l}0.52(0.35- \\
0.76)\end{array}$ & $\begin{array}{l}0.46(0.26- \\
0.83)^{\star}\end{array}$ \\
\hline & Other $* \star \star$ & $\begin{array}{l}1.61(0.95- \\
2.73)\end{array}$ & $\begin{array}{l}1.16(0.63- \\
2.14)\end{array}$ & $\begin{array}{l}0.81(0.47- \\
1.40)\end{array}$ & $\begin{array}{l}0.62(0.33- \\
1.17)\end{array}$ \\
\hline \multirow[t]{2}{*}{$\begin{array}{l}\text { Getting } \\
\text { information }\end{array}$} & yes & $\begin{array}{l}0.75(0.57- \\
0.98)\end{array}$ & $\begin{array}{l}0.02(0.05- \\
0.75)^{\star}\end{array}$ & $\begin{array}{l}0.58(0.44- \\
0.76)\end{array}$ & $\begin{array}{l}0.47(0.14- \\
1.61)\end{array}$ \\
\hline & no & Ref & Ref & Ref & Ref \\
\hline Source of & mass media & Ref & Ref & Ref & Ref \\
\hline
\end{tabular}




\begin{tabular}{|c|c|c|c|c|c|}
\hline \multirow{4}{*}{ information } & magazine & $\begin{array}{l}1.5(0.67- \\
3.34)\end{array}$ & $\begin{array}{l}1.43(0.18- \\
11.55)\end{array}$ & $\begin{array}{l}1.4(0.61- \\
3.23)\end{array}$ & $\begin{array}{l}1.43(0.17- \\
11.55)\end{array}$ \\
\hline & health institution & $\begin{array}{l}0.5(0.25- \\
1.01)\end{array}$ & $\begin{array}{l}0.44(0.20- \\
0.90)^{*}\end{array}$ & $\begin{array}{l}0.36(0.19- \\
0.68)\end{array}$ & $\begin{array}{l}1.94(0.23- \\
16.36)\end{array}$ \\
\hline & other people** & $\begin{array}{l}1.20(0.69- \\
2.11)\end{array}$ & $\begin{array}{l}1.72(0.23- \\
13.11)\end{array}$ & $\begin{array}{l}0.84(0.48- \\
1.46)\end{array}$ & $\begin{array}{l}0.75(0.09- \\
5.88)\end{array}$ \\
\hline & $\begin{array}{l}\text { Religious place } \\
\text { and school }\end{array}$ & $\begin{array}{l}1.34(0.77- \\
2.36)\end{array}$ & $\begin{array}{l}1.59(0.15- \\
16.98)\end{array}$ & $\begin{array}{l}1.28(0.73- \\
2.23)\end{array}$ & $\begin{array}{l}1.72(0.23- \\
13.10)\end{array}$ \\
\hline \multirow[t]{2}{*}{$\begin{array}{l}\text { Mental illness } \\
\text { history }\end{array}$} & yes & $\begin{array}{l}1.03(0.66- \\
1.61)\end{array}$ & $\begin{array}{l}0.99(0.62- \\
1.62)\end{array}$ & $\begin{array}{l}0.62(0.38- \\
0.98)\end{array}$ & $\begin{array}{l}1.78(1.05- \\
3.0)^{*}\end{array}$ \\
\hline & no & Ref & Ref & Ref & Ref \\
\hline \multirow[t]{4}{*}{$\begin{array}{l}\text { Relationship } \\
\text { with PWMI }\end{array}$} & relatives & $\begin{array}{l}1.09(0.64- \\
1.85)\end{array}$ & $\begin{array}{l}1.12(0.61- \\
2.04)\end{array}$ & $\begin{array}{l}1.12(0.67- \\
1.88)\end{array}$ & $\begin{array}{l}0.91(0.62- \\
1.35)\end{array}$ \\
\hline & neighbour & $\begin{array}{l}1.19(0.81- \\
1.75)\end{array}$ & $\begin{array}{l}1.11(0.73- \\
1.67)\end{array}$ & $\begin{array}{l}1.84(1.23- \\
2.74)\end{array}$ & $\begin{array}{l}1.54(0.92- \\
2.58)\end{array}$ \\
\hline & friend & $\begin{array}{l}1.56(1.08- \\
2.26)\end{array}$ & $\begin{array}{l}1.54(1.04- \\
2.28)^{\star}\end{array}$ & $\begin{array}{l}2.37(1.58- \\
3.55)\end{array}$ & $\begin{array}{l}2.75(1.70- \\
4.45)^{\star \star}\end{array}$ \\
\hline & Other* & Ref & Ref & Ref & Ref \\
\hline \multirow[t]{2}{*}{$\begin{array}{l}\text { Caring mentally } \\
\text { ill }\end{array}$} & yes & $\begin{array}{l}1.68(1.27- \\
2.23)\end{array}$ & $\begin{array}{l}1.71(1.25- \\
2.3)^{\star \star}\end{array}$ & $\begin{array}{l}0.45(0.34- \\
0.62)\end{array}$ & $\begin{array}{l}1.85(1.28- \\
2.65)^{\star \star}\end{array}$ \\
\hline & no & Ref & & Ref & Ref \\
\hline
\end{tabular}

PWMI- people with mental illness, ${ }^{*} p<0.05 * \star p<0.01$ ref $=$ reference. ${ }^{*}$ other $=$ on street, other people's home, ${ }^{* \star}$ other people $=$ informed by somewhere, ${ }^{* \star *}$ other $=$ jobless, retired and daily laborer

\section{Discussion}

This study assessed the community attitude to mental illness through four subscales. The four subscales of attitude measurement were authoritarianism, benevolence, social restrictiveness, and community mental health ideology.

The community held an avoidant attitude; they cannot go back to work, they are dangerous and should be treated far from the community residential area. This is supported by a community study done in Nigeria and Malaysia. The community believes that mentally ill people are mentally retarded, public nuisance and dangerous $(9,23)$. Many participants of the focus group discussion also support this; 'mentally ill peoples cannot control themselves and choose what is good for them rather act in contradiction. They shout, wander around the street, and bite people. Another participant said that mentally ill peoples act as 'brainless more than animals'. These ideas collectively describe how mentally ill people are stigmatized and discriminated by their community. The community called 'mentally ill' only 
those who are aggressive, violent and those who wander on the street. Due to the name given to the violent, all mentally ill patients are thought to be also violent and aggressive.

Age was significantly associated factor for authoritarianism, benevolence and social restrictiveness but not for mental health ideology. It has a positive association that showed older people are more authoritarian, benevolent and socially restrictive as compared to the youngsters. This is supported by a study finding in Malawi so that older peoples are more authoritarian and socially restrictive (24). Age was not associated with attitude on a study done in worabe town, silte zone, Ethiopia (18), a study done in Hawassa town (21) and community study done in Nigeria (9). The difference may be due to model difference, instrument and method difference. Elders were more authoritarian who force others to accept their ideas and consider patients inferior, more benevolent who strives to help mentally ill people and more socially restrictive affecting the social relationship of the patient. These authoritarian and socially restrictors are affecting people who have mental illness negatively because they do not allow them to take their choice, and to interact socially.

When people's educational status upgrades from elementary school to diploma and degree, their attitude of authoritarianism and benevolence decreases. This might be due to the fragmented information they could get from schooling and they are going to be less controlling and more negligent for the care of the mentally ill. Findings in Ghana show; when the educational status of the participant increases, their authoritarianism and social restrictiveness decrease (16). This study shows that when people learn more, their attitude to mental illness got more positive. This difference might be due to the population difference (all urban and mentally ill patients) and the way of using the tool (CAMI). The Ghanaian researcher used the tool as yes /no questionnaire and assessed the community attitude.

Farmers had negative mental health ideology as compared to government employees. This idea is similar to one focus group discussion participant's idea that states as follows: (F-9 is 26 years old, married merchant and knows mentally ill on the street) 'I don't have any information about mental illness but I know they are called mad (ebid). It is not helpful to take them anywhere because it is caused by God's punishment and evil spirit possession. Mostly they are left on the street.'

People who had information about mental illness were 4.18(1.07-16.26) times more authoritarian and their attitude of benevolent decreased by $78 \%$ as compared to those who do not. When people got more information, they are going to be more undermining and more un-humanistic to people who have mental illness. These types of people are more likely to keep mentally ill peoples behind a locked door.

Getting information from health institutions has a significant association with social restrictiveness [0.44 $\mathrm{Cl}(0.2-0.4) \mathrm{p}$-value $=0.03$ ] so that people were more supportive to people who have mental illness as compared to those who get information from mass media. This is supported by a study done in Hawasa town, Ethiopia: People who were informed by health workers were more comfortable giving a job and responsibility to people who have a mental illness (21). 
Peoples who have ever been hurt by the mentally ill were less benevolent than those who are not [AOR = $0.71(0.52-0.95) p$-value $=0.02]$. This is typical of focus group discussion participant's ideas and other researches that are done in Nigeria (9). People who have mental illness are thought to be more violent, nuisance and dangerous which directly affects the negative attitude positively. Female (F-1): I have been bitten by one mad man and after that I always afraid of them. They shall be left on the street because of their dangerous behavior. They are less likely to show improvement with treatment so that investing in people with mental illness is just wasting money. They are left on the street and their basic needs will not be met (15). This could expose mentally ill people to be prone to another medical problem because they are forced to live without human basic needs.

People who have friends with mental illness and people who have ever involved in caring for people with mental illness were more likely to accept mental health services and allow the establishment of mental health facilities to the local area of residency. This is about twice as likely as their counters and these ideas are interrelated to each other. Someone whose friend has mental illness is more likely to involve in caring for the victims and in turn, is more likely to demand mental health facilities around their residency area. They are also less likely to fear to think about establishing mental health facility in their area. These variables did not show association in a study done in worabe town, Ethiopia (18).

\section{Conclusion And Recommendation}

The findings of this study indicated that more than half of the community was more authoritarian, less benevolent, less socially restrictive and have a positive attitude to mental health-care service. People with mental illness were viewed as inferior and seen as who need supervision as Children. The community denied them emphatic and humanistic care. They were also denied to have a job and responsibility at all in the community. Getting information from health institutions had brought a positive attitude towards someone who has a mental illness. Public mental health awareness creating programs, rehabilitation centers for people with mental illness and public groups, like school clubs, which help people with mental illness, are major areas of tackling the bad attitude of the community. Using health institutions as a means of delivering mental health information is also important.

\section{Limitation of the study}

The limitation of this study might be the name mental illness is broad and lacks specificity.

\section{Acronyms And Abbreviations}

AMSH: Amanuel Mental Specialized Hospital AU: authoritarianism BE: benevolence CAMI: Community Attitude to Mental IIIness Inventory CMHI: community mental health ideology Epi info: Epidemiological Information GHSQ: General Help-Seeking Questionnaire MOH: Ministry of Health SPSS: Statistical Package for Social Science SR: social restrictiveness UOG: university of Gondar WHO: World Health Organization 


\section{Declarations}

\section{Ethical approval and consent to participate}

Ethical clearance was obtained from the joint review committee of the University of Gondar and Amanuel mental specialized hospital. Data collectors had explained the aim of the study and had asked oral consent before they started data collection. The right to ask a question about the study and to terminate the interview whenever they want to stop was offered for the study participants. Permission paper from the town administration was requested and distributed to the two kebelle administrations.

\section{Consent for publication}

Not applicable

\section{Availability of data and material}

The data sets used and/or analyzed during the current study are available from the corresponding authors on reasonable request.

\section{Authors' contributions}

$B Y, Z Y, S S$ involved in designing and conducting the study. BY, ZY and $A B$ involved in data collection, analysis, and interpretation of data. BY, ZY, SS, DA, and AB contributed to the designing and write up of the manuscript. All authors read and approved the final manuscript.

\section{Acknowledgment}

Our heartfelt thanks are to AMSH and the University of Gondar for the financial support and for giving us the opportunity to undertake this study. We are also highly indebted to thank the town community, data collectors, participants and town administration bodies for their cooperation. Finally, we want to acknowledge Wubanchi Sinshaw(S/r) for her help throughout the study period.

\section{Competing interest}

The authors declare that they have no competing interests.

\section{Funding}

Funding for this study was provided by a joint program from the University of Gondar and AMSH.

\section{References}

1. Organization WH. Mental health action plan 2013-2020. 2013.

2. Manderscheid RW, Ryff CD, Freeman EJ, McKnight-Eily LR, Dhingra S, Strine TW. Peer reviewed: evolving definitions of mental illness and wellness. Preventing chronic disease. 2010;7(1). 
3. Whiteford HA, Ferrari AJ, Degenhardt L, Feigin V, Vos T. Global burden of mental, neurological, and substance use disorders: an analysis from the Global Burden of Disease Study 2010. Mental, Neurological, and Substance Use Disorders. 2015:29.

4. Funk M. Global burden of mental disorders and the need for a comprehensive, coordinated response from health and social sectors at the country level. Retrieved on. 2016;30.

5. Health FDRoEMo. National Mental Health Strategy 2012/13-2015/16. Addis Ababa: Ministry of Health Addis Ababa; 2012.

6. Tibebe A, Tesfay K. Public knowledge and beliefs about mental disorders in developing countries: A review. Journal of Depression and Anxiety S. 2015;3:1-4.

7. Jorm AF. Mental health literacy: Public knowledge and beliefs about mental disorders. The British Journal of Psychiatry. 2000;177(5):396-401.

8. Henderson C, Robinson E, Evans-Lacko S, Thornicroft G. Relationships between anti-stigma programme awareness, disclosure comfort and intended help-seeking regarding a mental health problem. The British Journal of Psychiatry. 2017;211(5):316-22.

9. Gureje O, Lasebikan VO, Ephraim-Oluwanuga O, Olley BO, Kola L. Community study of knowledge of and attitude to mental illness in Nigeria. The British Journal of Psychiatry. 2005;186(5):436-41.

10. Kabir M, Iliyasu Z, Abubakar IS, Aliyu MH. Perception and beliefs about mental illness among adults in Karfi village, northern Nigeria. BMC International Health and Human Rights. 2004;4(1):3.

11. Ganesh K. Knowledge and attitude of mental illness among general public of Southern India. National journal of community medicine. 2011;2(1):175-8.

12. Girma E, Tesfaye M, Froeschl G, Möller-Leimkühler AM, Müller N, Dehning S. Public stigma against people with mental illness in the Gilgel Gibe Field Research Center (GGFRC) in Southwest Ethiopia. PloS one. 2013;8(12).

13. Hugo CJ, Boshoff DE, Traut A, Zungu-Dirwayi N, Stein DJ. Community attitudes toward and knowledge of mental illness in South Africa. Social psychiatry and psychiatric epidemiology. 2003;38(12):715-9.

14. Hailemariam KW. Perceived causes of mental illness and treatment seeking behaviors among people with mental health problems in Gebremenfes Kidus Holy Water Site. Am J Appl Psychol. 2015;3(2):34-42.

15. Teferra S, Shibre T. Perceived causes of severe mental disturbance and preferred interventions by the Borana semi-nomadic population in southern Ethiopia: a qualitative study. BMC psychiatry. 2012;12(1):79.

16. Barke A, Nyarko S, Klecha D. The stigma of mental illness in Southern Ghana: attitudes of the urban population and patients' views. Social psychiatry and psychiatric epidemiology. 2011;46(11):1191202.

17. Deribew A, Tamirat YS. How are mental health problems perceivedby a community in Agaro town? Ethiopian Journal of Health Development. 2005;19(2):153-9. 
18. Bedaso A, Yeneabat T, Yohannis Z, Bedasso K, Feyera F. Community attitude and associated factors towards people with mental illness among residents of Worabe Town, Silte Zone, southern nation's nationalities and people's region, Ethiopia. PloS one. 2016;11(3).

19. Reta Y, Tesfaye M, Girma E, Dehning S, Adorjan K. Public stigma against people with mental illness in Jimma Town, Southwest Ethiopia. PloS one. 2016;11(11).

20. Benti M, Ebrahim J, Awoke T, Yohannis Z, Bedaso A. Community perception towards mental illness among residents of Gimbi town, Western Ethiopia. Psychiatry journal. 2016;2016.

21. Ayano G, Agidew M, Duko B, Mulat H, Alemayew M. Perception, Attitude and Associated Factors on Schizophrenia and Depression Among Residents of Hawassa City, South Ethiopia, Cross Sectional Study. American Journal of Psychiatry and Neuroscience. 2015;3:116-24.

22. Bedaso A, Yeneabat T, Yohannis Z, Bedasso K, Feyera F. Community Attitude and Associated Factors towards People with Mental Illness among Residents of Worabe Town, Silte Zone, Southern Nation's Nationalities and People's Region, Ethiopia. PloS one. 2016;11(3):e0149429.

23. Yeap R, Low W. Mental health knowledge, attitude and help-seeking tendency: a Malaysian context. Singapore Med J. 2009;50(12):1169-76.

24. Jim Crabb1, 3*, , Robert C Stewart2, Demoubly Kokota2, Neil Masson2, 4, , Chabunya2 S, Krishnadas5 R. Attitudes towards mental illness in Malawi, a cross-sectional survey. BMC Public Health 2012;12(541). 